\title{
Back or neck-pain-related disability of nursing staff in hospitals, nursing homes and home care in seven countries- results from the European NEXT-Study
}

\author{
Michael Simon $^{\mathrm{a}, *}$, Peter Tackenberg ${ }^{\mathrm{a}}$, Albert Nienhaus ${ }^{\mathrm{b}}$, \\ Madeleine Estryn-Behar ${ }^{c}$, Paul Maurice Conway ${ }^{\text {, }}$, H.-M. Hasselhorn ${ }^{\mathrm{e}}$ \\ ${ }^{a}$ Department of Safety Engineering, Section of Occupational Safety \& Ergonomics, University of Wuppertal, Germany \\ ${ }^{\mathrm{b}}$ Berufsgenossenschaft für Gesundheitsdienst und Wohlfahrtspflege (BGW), Germany \\ ${ }^{\mathrm{c}}$ Assistance Publique-Hôpitaux de Paris, France \\ ${ }^{\mathrm{d}}$ Department of Occupational Health, University of Milan, Italy \\ ${ }^{\mathrm{e}}$ Department of Safety Engineering, Section of Occupational Health, University of Wuppertal, Germany
}

Received 19 April 2006; received in revised form 13 November 2006; accepted 14 November 2006

\begin{abstract}
Background: Musculoskeletal disorders are a widespread affliction in the nursing profession. Back or neck-pain-related disability of nursing staff is mainly attributed to physical and psychosocial risk factors.

Objectives: To investigate which — and to what extent—physical and psychosocial risk factors are associated with neck/ back-pain-related disability in nursing, and to assess the role of the type of health care institution (hospitals, nursing homes and home care institutions) within different countries in this problem.

Design: Cross-sectional secondary analysis of multinational data of nurses and auxiliary staff in hospitals $(n=16,770)$, nursing homes $(n=2140)$ and home care institutions $(n=2606)$ in seven countries from the European NEXT-Study. Methods: Multinomial logistic regression analysis with raw models for each factor and mutually adjusted with all analysed variables.

Results: Analysis of the pooled data revealed effort-reward imbalance as the predominant risk factor for disability in all settings (odds ratios for high disability by effort-reward ratio: hospital 5.05 [4.30-5.93]; nursing home 6.52 [4.04-10.52] and home care 6.4 [3.83-10.70] [after mutual adjustment of psychosocial and physical risk factors]). In contrast, physical exposure to lifting and bending showed only limited associations with odds ratios below 1.6; the availability and use of lifting aids was - after mutual adjustment - not or only marginally associated with disability. These findings were basically confirmed in separate analyses for all seven countries and types of institutions.

Conclusions: The findings show a pronounced association between psychosocial factors and back or neck-pain-related disability. Further research should consider psychosocial factors and should take the setting where nurses work into account.
\end{abstract}

(C) 2006 Elsevier Ltd. All rights reserved.

Keywords: Nursing staff; Hospital; Nursing homes; Home care; Back pain; Neck pain; Disability; Effort reward imbalance; Stress; International comparison; Belgium; Germany; France; Italy; The Netherlands; Poland; Slovakia

\footnotetext{
*Corresponding author. Tel.: + 492024393321.

E-mail address: sinext@uni-wuppertal.de (M. Simon).
} 


\section{What is already known about the topic?}

- Back or neck pain (and the related disability) is a common problem in nursing.

- Physical and psychosocial risk factors contribute to back or neck-pain-related disability.

- The working environment determines the physical and psychosocial exposure for nurses.

\section{What this paper adds}

- Effort reward imbalance (ERI) is a predominant risk factor in association with back or neck-pain-related disability.

- Neither lifting and bending nor the availability or usage of technical lifting aids was consistently associated with back or neck-pain-related disability.

- Both country and type of institution determine the risk factor pattern associated with disability.

\section{Background}

Back or neck pain is a health problem that affects all types of occupational groups. One third of all employees report work-related back pain (Paoli and Merllié, 2001). Despite this obviously widespread adversity, many occupational health researchers have focussed on the nursing profession, seen as a physically and psychosocially demanding profession with high prevalence rates of back-related complaints (Menzel, 2004). Besides the personal suffering, back pain is a major cause for healthrelated absenteeism. In Germany for example, data from a major health insurance company indicate that $56 \%$ of all reported sick days of nurses in in-patient units are due to musculoskeletal diseases and thus constitute the most reported cause for sick leave (Grabbe et al., 2005). Similar rates regarding the consequences of back pain have been reported in the USA (Panel on Musculoskeletal Disorders and the Workplace - Commission on Behavioral and Social Sciences and EducationNational Research Council, 2001), the UK (Smedley et al., 2003) and the Netherlands (Ijzelenberg and Burdorf, 2004). Finally, back complaints are recognised - in the long run - as a leading cause for early retirement (Pattani et al., 2001) which in some regions may worsen the nursing shortage.

Diverse models with various foci have been introduced to account for the development of musculoskeletal disorders (MSD). Bongers and colleagues (1993) for example have emphasised the link between psychosocial factors at work and musculoskeletal disease, Armstrong et al. (1993) developed a conceptual model for work-related neck and upper-limb disorders and the
'Panel on Musculoskeletal Disorders and the Workplace (2001) proposed a broad conceptual model, which covers workplace-related factors like physical load, the organisational and social context, personal factors such as biomechanical loading, internal tolerances (including psychological states) and several outcomes such as pain, discomfort and disability for different localisations. The link between psychosocial factors and MSD has often been analysed and discussed (Carayon et al., 1999, Davis and Heaney, 2000, Gunnarsdottir et al., 2003, Rugulies et al., 2004, Violante et al., 2004). Nowadays, it is widely accepted that both physical (e.g., lifting and bending) and psychological exposure (e.g., stress at work) at work are associated with back pain and disability.

To measure complaints related to MSD, both pain and disability are outcomes used (Elders and Burdorf, 2001). Like intensity or recency of onset of complaints, disability is one of several indicators in the global assessment of pain (von Korff et al., 1992). However, in contrast to other pain measurements, disability is based on functional restrictions initiated through pain (Turner et al., 2004) and can be regarded as a consequence of pain.

Although the nursing profession is established in different parts of the health care system, studies often do not differentiate between different qualification levels of nurses and types of institutions where nurses work. In addition nurses' work and the working conditions vary across countries. To address this ecological background we summarise the two dimensions: country and type of institutions with the term 'setting'. While it seems to be unlikely that different settings cause different mechanisms for the development of MSD and disability, it seems to be inappropriate to ignore the setting. In their review on MSD, Sherehiy et al. (2004) identified only a few studies differentiating between groups of nurses according to (for example) qualification level (aides vs. registered nurses) and settings (hospital, nursing homes and homecare). Although back and neck pain and to a lower extent disability due to back and neck pain in nursing in hospitals and nursing homes have been extensively investigated, there is little research on nursing homes and home care. Cheung et al. (2006) for example identified only seven studies investigating home care settings. Although this might underestimate the number of research studies in this field, research comparing back or neck-related complaints in different nursing settings is still uncommon.

In summary, we conclude that neck or back-painrelated disability is a relevant issue in nursing, that both psychosocial and physical factors are contributing to back or neck-related complaints and that 'back and neck-pain-related disability' may constitute a valid alternative measure to the assessment of pain. Finally only little attention has been put on the influence of the setting with respect to MSD in nursing. 


\section{Objectives}

The data that were analysed stem from the European NEXT-Study and allows:

(a) the investigation of which - and to what extentphysical and psychosocial risk factors are associated with neck/back-pain-related disability in nursing, and

(b) the assessment of the role of the type of health care institution (hospitals, nursing homes and home care institutions) overall and in the countries investigated.

\section{Data}

The 'Nurses' early exit study' (NEXT) is a European research project investigating premature departure from the nursing profession (for general information: http:// www.next-study.net). More than 56,000 health care workers from 11 countries have participated in crosssectional and prospective questionnaire assessments. Separately for each country, quota samples were drawn that were intended to cover the main working areas (hospitals, nursing homes, and home care) and the national geographical distribution of nurses. More information on the aims, design, and performance of the NEXT-Study can be found in Hasselhorn et al. (2003, 2005). Ethical approval was given by the ethical committee of the University of Wuppertal (Germany).

In this paper, data from the basic questionnaire assessment 2002/2003 was used. The analysis was limited to data from seven participating European countries (Belgium, Germany, France, Italy, the Netherlands, Poland, Slovakia) and to nursing aides with at least one year of training as well as registered nurses (meeting the educational requirements of the Sectoral Directive of the European Union). Of 30,617 respondents a total of
21,516 participants with valid responses to all items were selected and used in the analysis. Following the study objectives, sub samples for hospitals $(n=16,770)$, nursing homes $(n=2140)$ and home care institutions $(n=2606)$ were created (Table 1$)$.

\section{Methods}

For all scales used in the analysis and described below satisfactory psychometric properties have been found (Kümmerling et al., 2003).

\subsection{Outcome measurement}

Disability was measured using a four-item-scale developed by von Korff et al. (1992) covering both neck and/or low back pain in relation to days being 'disabled' in the past six months and interference with daily activities, social activities and the ability to work. In NEXT, the scales for disability due to neck/shoulder and due to low back pain were merged, (e.g., 'how much has neck or low back pain interfered with your daily activities'). The calculated disability score ranged from 0 to 6 , which then was classified into 0 'no disability', 1 to 3 'moderate disability' and 4 to 6 'high disability'. This trichotomisation was used as outcome variable, with 'no disability' cases as reference groups.

\subsection{Physical factors}

Physical risk factors were assessed with two measures: the 'lifting and bending' score (L\&B) and the availability and usage of technical lifting aids. L\&B consists of an 8item scale developed by the NEXT-Study Group covering the frequency of characteristic physically demanding tasks of nursing work, in particular patient-handling activities such as getting patients to bed, and positioning and lifting of patients (Kümmerling et al., 2003). The scores of each item were weighed and a sum score

Table 1

Sample by country

\begin{tabular}{|c|c|c|c|c|c|c|}
\hline \multirow[t]{2}{*}{ Country } & \multicolumn{2}{|c|}{ Basic questionnaires } & \multirow[t]{2}{*}{ Response rate $(\%)$} & \multicolumn{3}{|c|}{ Analyzed sample } \\
\hline & Sent out & Received & & Hospital & Nursing homes & Home care \\
\hline Belgium & 7049 & 4257 & 60.4 & 1819 & 208 & 1253 \\
\hline Germany & 6484 & 3565 & 55.0 & 2181 & 346 & 256 \\
\hline France & 13,017 & 5376 & 41.3 & 3043 & 397 & 45 \\
\hline Italy & 7447 & 5645 & 75.8 & 3478 & 124 & 285 \\
\hline Netherlands & 9309 & 4024 & 43.2 & 2338 & 673 & 573 \\
\hline Poland & 7091 & 4354 & 61.4 & 2520 & 138 & 161 \\
\hline \multirow[t]{2}{*}{ Slovakia } & 6382 & 3396 & 53.2 & 1391 & 254 & 33 \\
\hline & 56,779 & 30,617 & 53.9 & 16,770 & 2140 & 2606 \\
\hline
\end{tabular}


ranging from 0 to 100 was set. For the regression analysis L\&B was categorised into tertiles separately according to type of institution. The lowest tertile 'low physical exposure' constitutes the reference category in the logistic regression analysis. Also for analysis on country level, the tertile ranges defined for the total sample were used.

Availability and usage of technical lifting aids was assessed by a filter question resulting in three possible categories: 'lifting aids not available', 'lifting aids available but not used' and, finally 'lifting aids available and used' (reference category).

\subsection{Psychosocial factors}

As indicators for psychosocial risk factors the following three concepts were used: 'quantitative demands', 'influence at work' and the 'effort-reward imbalance ratio' (ERI ratio). Quantitative demands indicating work intensity were measured using a five-item scale based on the Copenhagen Psychosocial Questionnaire (COPSOQ, Kristensen et al., 2005) and supplemented by a nursing specific item. The scale was trichotomised (separately by type of institution) with the lowest tertile as reference group. The four-item scale 'influence at work' indicating control over one's working environment derives from the Swedish version of the Demand-Control questionnaire (Theorell et al., 1988) and was adopted by the NEXT-Study Group in an adjusted form (Kümmerling et al., 2003). Following trichotomisation (again by type of institution) the highest tertile constitutes the reference group indicating highest influence at work. The ERI ratio measure constitutes the core of the effort-reward imbalance model developed by Siegrist (Siegrist, 1996). It is based on the postulate that 'digression from reciprocity in transaction results in a stressful experience'. Applied to the workplace, this would mean that there should be a balance between what the employee gives ('effort') and what he or she receives ('reward'). Here, reward not only includes financial reward but also esteem and career opportunities. The short version of the ERI questionnaire was used (Siegrist et al., 2004). The relation between the individual's effort and reward scores is calculated as the ERI ratio, theoretically ranging from .2 to 5 . Trichotomisation (by type of institution) resulted in three tertiles with the lowest indicating a fortunate ERI ratio (reference category), which means a balance between efforts and reward (low stress), and the highest with a high imbalance between efforts and perceived rewards at the workplace (high stress). Again, analysis on country level was performed on the basis of the tertile ranges defined for the total sample.

\subsection{Individual factors}

Several reviews (Lagerstrom et al., 1998, Menzel, 2004, Sherehiy et al., 2004) have shown that qualifica- tion level and therewith associated different exposure and tasks are associated with the risk for spine complaints. Consequently, the distinction between nurses and auxiliary staff was used as separate risk factor, with registered nurses as the reference category.

\subsection{Statistical methods}

For estimation of risk factors of back-pain-related disability, multinomial logistic regressions were performed. Multinomial regression is used when the outcome is polytomous and delivers the odds of exposure among those belonging to a specific category of the multicategorial outcome. In our study, the moderate disability (1-3) and the severe disability group (4-6) were compared with the odds of exposure among those belonging to the reference group, the non-disability group. For each type of institution two models were computed. Model (a) consists of each of the assumed risk factors (e.g., lifting and bending) and a fixed set of control variables: gender, age and country. This adjustment was chosen to prevent effects from the unequal distribution of gender and age in the different institution types and to adjust for national features potentially influencing the data from the different countries. Model (b) combined all risk factors and control variables and delivered mutually adjusted odds ratios. In addition, separate analysis by country and type of institution were performed. For reasons of comprehensibility, we reported only the associations between exposure and the high disability category, and this was only done for model B (mutual adjustment and adjustment for age; we did not adjust for gender, because too many empty cells emerged due to few male nurses). All statistical tests were conducted using SPSS version 11 (SPSS Inc., 2005).

\section{Results}

\subsection{Descriptive analysis}

Descriptive analysis (Table 2) indicates that the proportion of nursing staff without disability is highest in home care $(62.3 \%)$ and somewhat lower in hospitals $(53.8 \%)$ and nursing homes $(52.0 \%, \mathrm{NH})$. As expected $\mathrm{NH}$ had the highest rate of respondents with high exposure to L\&B $(63.5 \%)$. Respondents in hospitals reported the lowest availability of lifting aids (in $62.9 \%$ not available) and the highest mean ERI ratio indicating a higher adverse imbalance between effort and rewards at work. In home care institutions the conditions assessed were least adverse in comparison to both inpatient settings. 
Table 2

Sample by type of institution through gender, qualification level, prevalence of disability, availability and usage of technical lifting aids

\begin{tabular}{|c|c|c|c|c|c|c|}
\hline & \multicolumn{2}{|c|}{ Hospital (\%) } & \multicolumn{2}{|c|}{ Nursing home $(\%)$} & \multicolumn{2}{|c|}{ Home care $(\%)$} \\
\hline Female & 85.8 & & 92.2 & & 95.1 & \\
\hline Male & 14.3 & & 7.8 & & 4.9 & \\
\hline Age $16+$ & 17.6 & & 18.6 & & 14.9 & \\
\hline Age $30+$ & 57.4 & & 48.2 & & 53.5 & \\
\hline Age $45+$ & 25.0 & & 33.2 & & 31.7 & \\
\hline RNs & 93.0 & & 70.1 & & 89.6 & \\
\hline Aids & 7.0 & & 30.0 & & 10.4 & \\
\hline No disability & 53.8 & & 52.0 & & 62.3 & \\
\hline Medium disability & 33.5 & & 33.2 & & 28.7 & \\
\hline High disability & 12.7 & & 14.8 & & 9.1 & \\
\hline Technical lifting aids: not available & 62.9 & & 22.4 & & 36.3 & \\
\hline Technical lifting aids: not used & 12.4 & & 16.2 & & 3.7 & \\
\hline \multirow[t]{2}{*}{ Technical lifting aids: used } & 24.8 & & 61.4 & & 60.0 & \\
\hline & Mean & Range & Mean & Range & Mean & Range \\
\hline L\&B (low) & 6.9 & $0-14.6$ & 10.8 & $0-20.4$ & 3.1 & $0-9.6$ \\
\hline L\&B (medium) & 23.0 & $15.4-31.7$ & 30.9 & $21.3-42.1$ & 16.8 & $11.7-22.9$ \\
\hline L\&B (high) & 53.2 & $32.5-100$ & 63.5 & $42.5-100$ & 34.8 & $23.3-87.5$ \\
\hline Quantitative demands (low) & 2.65 & $1.0-3.0$ & 2.60 & $1.0-3.0$ & 2.46 & $1.0-3.0$ \\
\hline Quantitative demands (medium) & 3.40 & $3.2-3.6$ & 3.39 & $3.2-3.6$ & 3.10 & $3.0-3.25$ \\
\hline Quantitative demands (high) & 4.06 & $3.75-5.0$ & 4.09 & $3.75-5.0$ & 3.73 & $3.4-5.0$ \\
\hline Influence at work (high) & 3.94 & $3.5-5.0$ & 3.95 & $3.5-5.0$ & 4.08 & $3.75-5.0$ \\
\hline Influence at work (medium) & 3.00 & $2.75-3.33$ & 3.00 & $2.75-3.33$ & 3.26 & $3.0-3.67$ \\
\hline Influence at work (low) & 2.07 & $1.0-2.67$ & 2.11 & $1.0-2.67$ & 2.33 & $1.0-2.75$ \\
\hline ERI (low) & .39 & $.20-.51$ & .35 & $.20-.44$ & .31 & $.20-.37$ \\
\hline ERI (medium) & .62 & $.51-.75$ & .56 & $.45-.67$ & .45 & $.37-.53$ \\
\hline ERI (high) & 1.07 & $.75-5.0$ & .98 & $.68-3.27$ & .77 & $.53-5.0$ \\
\hline$n$ & \multicolumn{2}{|c|}{16,770} & \multicolumn{2}{|c|}{2140} & \multicolumn{2}{|c|}{2606} \\
\hline
\end{tabular}

Mean scores for age, effort-reward imbalance ratio and degree of lifting and bending (L\&B).

\subsection{Risk factor analysis}

The multinomial logistic regression analysis confirms the association of the selected risk factors with back and neck-pain-related disability (Tables 3-4). Expectedly, higher exposure was associated with higher risks indicating an exposure-outcome gradient. Notably, for almost all variables the odds ratios (OR) were higher for the extreme 'high disability' classification and somewhat reduced OR for 'medium disability'. This was the case for all independent variables.

Risk factor analysis of different types of institutions showed similar patterns for in-patient facilities (hospitals and nursing homes) and to some extent diverging for home care institutions where OR were mostly lower.

As expected, nursing aides were slightly more at risk of disability. Interestingly, this increased after mutual adjustment (Model b) of all independent variables.

In all three institution types L\&B was associated with disability. Members of the group with highest lifting and bending exposure had - on bivariate level — an up to 2.5 fold higher risk than their colleagues with lower lifting exposure. Mutual adjustment of variables, however, reduced the OR considerably indicating a weak or limited association of L\&B and disability.

The availability and use of lifting aids in hospitals and in NH was only marginally associated with disability. There was no association in home care institutions. Quantitative demands were the second strongest predictor of disability (OR up to 3.3), however, mutual adjustment reduced the OR substantially. The association of 'influence at work' with disability (bivariate level OR up to 1.9) disappeared completely after mutual adjustment in all three institution types.

The strongest predictor of disability was by far the ERI ratio. The tertile of participants with the highest ERI ratio had in hospitals a 6.2 fold risk for suffering from disability, in nursing homes this was 7.3 and in home care institutions 8.1. After mutual adjustment the OR were still considerably high $(5.1,6.5$ and 6.4 , respectively).

The analysis by country and type of institution (Table 5) confirms these findings with a high degree of consistency, supporting a strong association between ERI and disability. In 14 of 19 settings ERI was significantly associated with disability, L\&B was sig- 
Table 3

Risk factors for disability: bivariate odds adjusted for age, gender \& country

\begin{tabular}{|c|c|c|c|c|c|c|c|c|c|c|c|c|}
\hline \multirow[t]{3}{*}{ Model A } & \multicolumn{4}{|c|}{ Hospitals } & \multicolumn{4}{|c|}{ Nursing homes } & \multicolumn{4}{|c|}{ Home care } \\
\hline & \multicolumn{2}{|c|}{ Medium disability } & \multicolumn{2}{|c|}{ High disability } & \multicolumn{2}{|c|}{ Medium disability } & \multicolumn{2}{|c|}{ High disability } & \multicolumn{2}{|c|}{ Medium disability } & \multicolumn{2}{|c|}{ High disability } \\
\hline & OR & $95 \% \mathrm{CI}$ & OR & $95 \% \mathrm{CI}$ & OR & $95 \% \mathrm{CI}$ & OR & $95 \% \mathrm{CI}$ & OR & $95 \% \mathrm{CI}$ & OR & $95 \% \mathrm{CI}$ \\
\hline Qualification & - & - & - & - & - & - & - & - & - & - & - & - \\
\hline Qualification (aids) & $1.98 * *$ & $(1.61-2.43)$ & $1.85 * *$ & $(1.52-2.25)$ & 1.34 & $(1.05-1.72)$ & $1.82 * *$ & $(1.32-2.50)$ & 0.97 & $(0.68-1.37)$ & 1.22 & $(0.77-1.90)$ \\
\hline L\&B (low) & - & - & - & - & - & - & - & - & - & - & - & - \\
\hline L\&B (medium) & 1.12 & $(0.98-1.27)$ & $1.40 * *$ & $(1.24-1.59)$ & 1.23 & $(0.96-1.56)$ & $1.58^{* *}$ & $(1.12-2.24)$ & $1.36^{*}$ & $(1.04-1.77)$ & 1.36 & $(0.91-2.05)$ \\
\hline L\&B (high) & $1.35^{* *}$ & $(1.19-1.54)$ & $2.06 * *$ & $(1.83-2.33)$ & $1.79 *$ & $(1.39-2.29)$ & $2.44 * *$ & $(1.73-3.44)$ & $1.41^{*}$ & $(1.06-1.87)$ & $1.69^{*}$ & $(1.10-2.60)$ \\
\hline Lifting aids (available + used) & - & - & - & - & - & - & - & - & - & - & - & - \\
\hline Lifting aids (no) & $1.21^{* *}$ & $(1.06-1.39)$ & $1.15^{*}$ & $(1.01-1.31)$ & 0.91 & $(0.67-1.21)$ & 1.33 & $(0.92-1.92)$ & 1.09 & $(0.87-1.36)$ & 0.84 & $(0.59-1.18)$ \\
\hline Lifting aids (not used) & 1.20 & $(1.00-1.44)$ & $1.29 * *$ & $(1.08-1.54)$ & 1.07 & $(0.80-1.43)$ & 1.28 & $(0.89-1.84)$ & 1.14 & $(0.72-1.79)$ & 0.58 & $(0.25-1.34)$ \\
\hline Quantitative demands (low) & - & - & - & - & - & - & - & - & - & - & - & - \\
\hline Quantitative demands (med) & 1.07 & $(0.93-1.22)$ & $1.59 * *$ & $(1.40-1.81)$ & $1.33^{*}$ & $(1.05-1.69)$ & $2.11 * *$ & $(1.50-2.96)$ & 1.18 & $(0.93-1.51)$ & $1.66^{*}$ & $(1.09-2.52)$ \\
\hline Quantitative demands (high) & $1.52 * *$ & $(1.31-1.76)$ & $3.08 * *$ & $(2.71-3.49)$ & $1.84 * *$ & $(1.42-2.36)$ & $2.64 * *$ & $(1.85-3.78)$ & $1.74 * *$ & $(1.40-2.16)$ & $3.24 * *$ & $(2.27-4.63)$ \\
\hline Influence at work (high) & - & - & - & - & - & - & - & - & - & - & - & - \\
\hline Influence at work (med) & 0.91 & $(0.80-1.03)$ & $1.14^{*}$ & $(1.01-1.29)$ & 1.26 & $(1.00-1.59)$ & $1.44^{*}$ & $(1.04-1.99)$ & 1.23 & $(0.99-1.53)$ & 1.22 & $(0.86-1.73)$ \\
\hline Influence at work (low) & 0.95 & $(0.83-1.08)$ & $1.55^{* *}$ & $(1.38-1.75)$ & $1.60 * *$ & $(1.25-2.05)$ & $1.91 * *$ & $(1.38-2.66)$ & $1.64 * *$ & $(1.30-2.06)$ & $1.49^{*}$ & $(1.04-2.14)$ \\
\hline ERI (low) & - & - & - & - & - & - & - & - & - & - & - & - \\
\hline ERI (med) & $1.97 *$ & $(1.69-2.31)$ & $2.14 * *$ & $(1.84-2.49)$ & $1.63 * *$ & $(2.40-4.26)$ & $1.93 * *$ & $(1.24-2.98)$ & $1.74 * *$ & $(1.37-2.20)$ & $3.49 * *$ & $(2.14-5.67)$ \\
\hline ERI (high) & $5.05^{* *}$ & $(4.30-5.93)$ & $6.20 * *$ & $(5.36-7.16)$ & $3.20 * *$ & $(1.27-2.10)$ & $7.25 * *$ & $(4.69-11.20)$ & $3.19 * *$ & $(2.50-4.06)$ & $8.07^{* *}$ & $(5.00-13.04)$ \\
\hline
\end{tabular}

$* p<0.05, * * p<0.01$.

Table 4

Risk factors for disability: multivariate odds mutually adjusted including age, gender \& country

\begin{tabular}{|c|c|c|c|c|c|c|c|c|c|c|c|c|}
\hline \multirow[t]{3}{*}{ Model B } & \multicolumn{4}{|c|}{ Hospitals } & \multicolumn{4}{|c|}{ Nursing homes } & \multicolumn{4}{|c|}{ Home care } \\
\hline & \multicolumn{2}{|c|}{ Medium disability } & \multicolumn{2}{|c|}{ High disability } & \multicolumn{2}{|c|}{ Medium disability } & \multicolumn{2}{|c|}{ High disability } & \multicolumn{2}{|c|}{ Medium disability } & \multicolumn{2}{|c|}{ High disability } \\
\hline & OR & $95 \% \mathrm{CI}$ & OR & $95 \% \mathrm{CI}$ & OR & $95 \% \mathrm{CI}$ & OR & $95 \% \mathrm{CI}$ & OR & $95 \% \mathrm{CI}$ & OR & $95 \% \mathrm{CI}$ \\
\hline Qualification & - & - & - & - & - & - & - & - & - & - & - & - \\
\hline Qualification (aids) & $1.29 * *$ & $(1.10-1.50)$ & $1.98 * *$ & $(1.61-2.43)$ & $1.37 *$ & $(1.07-1.77)$ & $2.02 * *$ & $(1.43-2.83)$ & 1.08 & $(0.75-1.55)$ & 1.49 & $(0.92-2.44)$ \\
\hline L\&B (low) & - & - & - & - & - & - & - & - & - & - & - & - \\
\hline L\&B (medium) & $1.12 *$ & $(1.02-1.22)$ & 1.12 & $(0.98-1.27)$ & $1.41 *$ & $(1.08-1.84)$ & 1.20 & $(0.83-1.73)$ & 1.31 & $(0.99-1.72)$ & 1.15 & $(0.74-1.76)$ \\
\hline L\&B (high) & $1.17^{* *}$ & $(1.07-1.28)$ & $1.35^{* *}$ & $(1.19-1.54)$ & 1.07 & $(0.82-1.38)$ & $1.59 * *$ & $(1.10-2.30)$ & 1.23 & $(0.91-1.66)$ & 1.21 & $(0.77-1.92)$ \\
\hline Lifting aids (available + used) & - & - & - & - & - & - & - & - & - & - & - & - \\
\hline Lifting aids (no) & 1.05 & $(0.96-1.15)$ & $1.21^{* *}$ & $(1.06-1.39)$ & 0.97 & $(0.72-1.32)$ & 1.48 & $(1.00-2.18)$ & 1.09 & $(0.87-1.38)$ & 0.87 & $(0.60-1.25)$ \\
\hline Lifting aids (not used) & $1.18^{*}$ & $(1.04-1.33)$ & 1.20 & $(1.00-1.44)$ & 1.01 & $(0.75-1.37)$ & 1.19 & $(0.81-1.75)$ & 0.95 & $(0.60-1.52)$ & 0.47 & $(0.20-1.10)$ \\
\hline Quantitative demands (low) & - & - & - & - & - & - & - & - & - & - & - & - \\
\hline Quantitative demands (med) & 0.98 & $(0.90-1.07)$ & 1.07 & $(0.93-1.22)$ & 1.08 & $(0.81-1.46)$ & 1.34 & $(0.93-1.95)$ & 0.95 & $(0.74-1.23)$ & 1.24 & $(0.80-1.92)$ \\
\hline Quantitative demands (high) & $1.17 * *$ & $(1.05-1.29)$ & $1.52 * *$ & $(1.31-1.76)$ & 1.02 & $(0.79-1.31)$ & 1.12 & $(0.74-1.70)$ & 1.09 & $(0.85-1.39)$ & $1.85^{* *}$ & $(1.24-2.78)$ \\
\hline Influence at work (high) & - & - & - & - & - & - & - & - & - & - & - & - \\
\hline Influence at work (med) & 0.99 & $(0.91-1.08)$ & 0.91 & $(0.80-1.03)$ & 1.14 & $(0.87-1.49)$ & 1.03 & $(0.73-1.46)$ & 1.07 & $(0.85-1.34)$ & 0.93 & $(0.64-1.34)$ \\
\hline Influence at work (low) & 1.02 & $(0.93-1.11)$ & 0.95 & $(0.83-1.08)$ & 1.04 & $(0.81-1.33)$ & 1.04 & $(0.72-1.50)$ & 1.24 & $(0.96-1.59)$ & 0.86 & $(0.58-1.28)$ \\
\hline ERI (low) & - & - & - & - & - & - & - & - & - & - & - & - \\
\hline ERI (med) & $1.79 * *$ & $(1.63-1.97)$ & $1.97 *$ & $(1.69-2.31)$ & $1.52 * *$ & $(2.05-3.91)$ & $1.77 * *$ & $(1.13-2.78)$ & $1.68 * *$ & $(1.32-2.15)$ & $3.08 * *$ & $(1.87-5.05)$ \\
\hline ERI (high) & $2.83 * *$ & $(2.55-3.15)$ & $5.05^{* *}$ & $(4.30-5.93)$ & $2.83^{* *}$ & $(1.17-1.99)$ & $6.52 * *$ & $(4.04-10.52)$ & $2.91 * *$ & $(2.23-3.80)$ & $6.40^{* *}$ & $(3.83-10.70)$ \\
\hline
\end{tabular}

$* p<0.05, * * p<0.01$.

nificant in 7 , quantitative demands in 6 and technical lifting aids in five settings (all findings in the expected direction).

\section{Discussion}

This study showed a pronounced relationship between psychosocial factors and back or neck-pain-related disability, which was higher than the association with physical factors. Furthermore this analysis showed that this finding was consistent across all types of institutions and qualification levels in all countries, in both crude and adjusted models.

This result raises mainly three questions regarding the first study objective, the investigation of the association of physical and psychosocial risk factors to neck/backpain-related disability: Are the results in line with 
previous research? Is disability more susceptible to psychosocial risk factors? And, finally, what are possible explanations for the weak association found between physical load and disability?

The association between psychosocial factors and MSD in general is still controversial. Different authors found no or weak relations between back pain or disability and psychosocial variables in both reviews (Davis and Heaney, 2000) and in primarily empirical papers (Hoogendoorn et al., 2002), whereas others found support for such an interrelation (Crook et al., 2002, van den Heuvel et al., 2004). The strong association of ERI-ratio and back-pain-related disability found in our investigation supports the role of psychosocial factors in association with back or neckpain-related disability. It is in line with two other investigations (van Vegchel et al., 2005) which linked musculoskeletal outcomes with the ERI-model, and our results are consistent with previous evidence of a significant association between psychosocial factors and both acute (often pain) and chronic states (disability) (Linton, 2000). Nevertheless it should be kept in mind, that this analysis is based on a cross-sectional survey and is therefore not qualified for drawing any causal inferences.

Regarding the unexpected weak association of $L \& B$ and disability, we have to point out, that measurements used in this secondary analysis were primarily focussed on the aims of the NEXT-Study, which are different from those of this analysis. We used combined 'back and neck-pain-related disability' as outcome, a measure that is less common than the direct assessment of pain and furthermore not specific regarding the location of the pain (low back, neck, upper limb, etc.). This lack of precision may have an impact on the association with the investigated factors, mainly leading to an underestimation of risk. The L\&B scale might have shown a higher association with back related disability compared to neck-related disability. We cannot exclude a stronger association in the assessment of low back disability only. Another reason may be the following: According to Turner et al. (2004) disability is especially related to more severe states of pain. Maybe physical exposure is more associated with less severe neck and low back outcomes and, in contrast, the role of psychosocial factors might increase with the severity of pain and therefore the more chronic states of disability. This supports the more pronounced association of disability with ERI in contrast to L\&B.

The dominant role of ERI and the major reduction of quantitative demands and influence at work in the mutually adjusted model (b) could be partly attributed to the theoretical background of the variables. Both quantitative demands and influence at work on the one hand and effort-reward imbalance on the other hand stems from stress models and may in parts explain the same phenomena. However correlation of ERI and quantitative demands (.49) and influence at work (.27) rule out multicollinearity as an explanation.

The rather weak association of physical factors (L\&B and availability and usage of lifting aids) raises the question about validity of the instruments we used. The L\&B score consists of eight items, which assessed the amount of relevant physical tasks like getting patients to bed, transferring and clothing patients, but also miscellaneous tasks such as making beds, pushing trolleys, etc. Content validity was repeatedly assessed by experts, scores for skewness and kurtosis were satisfactorily ranging between -.07 and 1.28 in all ten participating countries and the internal consistency of the scale was high (alpha values ranging from .79 to .91 [Kümmerling et al., 2003]). The range of our L\&B scale is from 0 to 100. A mean score of 50 already indicates very high physical loads. Our data, however, indicate that only after a measure of 60 a steep increase of disability occurs, but such a high physical load was rare in our nursing sample (only about $10 \%$ of all participants). We do not question that even a 'normal' physical load may lead to spine complaints, but we speculate that other influential factors (such as psychosocial work load and other health-related conditions) may hide this effect making it very difficult or even impossible to measure. Only extreme physical exposure leaves space for an association between physical exposure and measurements of spine complaints.

Regarding the second aim of the study, our results precisely document the heterogeneous nature of nurses' work across the different settings (types of institutions, countries). Higher exposure to L\&B in nursing homes, a pronounced effort-reward imbalance in hospitals, and lower exposures in both domains in home care institutions are consistent with common expectations for these types of organisations.

The results from this and another analysis (Simon et al., 2005) implicate that the type of institution (and the national background) mainly define the psychosocial and physical demands of nurses. Therefore differences regarding the institutional type should be considered as an important ecological variable.

\section{Conclusion}

The strong relation of psychosocial factors (especially effort and reward) and disability adds these to a list of factors that should be addressed by preventive measures for back or neck-pain-related disability. This supports the call for multifactor interventions (Hignett, 2003) and highlights the need for research on interventions covering the contribution of both psychosocial and physical factors. 
Table 5

Risk factors for high disability by type of institutions and country: multivariate odds mutually adjusted including age

\begin{tabular}{|c|c|c|c|c|c|c|c|}
\hline & Belgium & Germany & France & Italy & Netherlands & Poland & Slovakia \\
\hline & Hospital $(n=1819)$ & Hospital $(n=2181)$ & Hospital $(n=3043)$ & Hospital $(n=3478)$ & Hospital $(n=2338)$ & Hospital $(n=2520)$ & Hospital $(n=1391)$ \\
\hline & Odds $(95 \% \mathrm{CI})$ & Odds $(95 \% \mathrm{CI})$ & Odds $(95 \% \mathrm{CI})$ & Odds $(95 \% \mathrm{CI})$ & Odds $(95 \% \mathrm{CI})$ & Odds $(95 \% \mathrm{CI})$ & Odds $(95 \% \mathrm{CI})$ \\
\hline \multicolumn{8}{|l|}{ Qualification } \\
\hline Qualification (aids) & $2.16(1.32-3.55)^{* *}$ & $1.13(0.46-2.79)$ & $1.97(1.53-2.54)^{* *}$ & $1.02(1.01-1.04)^{* *}$ & $4.27(1.69-10.79)^{* * *}$ & (Only RN in sample) & (Only RN in sample) \\
\hline \multicolumn{8}{|l|}{ L\&B (low) } \\
\hline L\&B (medium) & $1.24(0.76-2.03)$ & $1.37(0.98-1.93)$ & $1.20(0.89-1.61)$ & $0.95(0.73-1.23)$ & $0.67(0.36-1.25)$ & $1.09(0.79-1.51)$ & $1.40(0.96-2.05)$ \\
\hline L\&B (high) & $1.59(1.00-2.52)^{*}$ & $1.48(1.04-2.11)^{*}$ & $1.05(0.79-1.41)$ & $0.93(0.72-1.20)$ & $1.05(0.58-1.87)$ & $1.56(1.14-2.15)^{*}$ & $2.34(1.58-3.47)^{* *}$ \\
\hline \multicolumn{8}{|c|}{ Lifting Aids (available + used) } \\
\hline Lifting aids $(\mathrm{n} / \mathrm{a})$ & $1.11(0.75-1.64)$ & $1.25(0.91-1.73)$ & $1.14(0.88-1.49)$ & $1.41(1.07-1.85)^{*}$ & $0.77(0.46-1.28)$ & $2.02(1.11-3.69)^{*}$ & $0.9(0.33-3.00)$ \\
\hline Lifting aids (not used) & $1.13(0.73-1.74)$ & $0.93(0.62-1.40)$ & $1.55(1.08-2.21)^{*}$ & $1.38(0.94-2.04)$ & $0.84(0.42-1.68)$ & $1.91(0.70-5.25)$ & $1.53(0.07-31.66)$ \\
\hline \multicolumn{8}{|l|}{ Quantitative demands (low) } \\
\hline Quantitative demands (med) & $0.83(0.53-1.31)$ & $1.19(0.81-1.75)$ & $0.85(0.63-1.13)$ & $1.35(1.02-1.79) *$ & $1.19(0.70-2.04)$ & $1.15(0.79-1.69)$ & $1.05(0.70-1.58)$ \\
\hline \multicolumn{7}{|c|}{ Influence at work (high) } & $1.74(1.13-2.69)^{* *}$ \\
\hline Influence at work (med) & $0.96(0.63-1.47)$ & $0.71(0.52-0.97)$ & $1.14(0.86-1.52)$ & $0.93(0.70-1.22)$ & $0.55(0.32-0.96)^{*}$ & $0.96(0.69-1.35)$ & $0.89(0.58-1.36)$ \\
\hline Influence at work (low) & $0.90(0.58-1.41)$ & $0.97(0.71-1.33)$ & $1.25(0.93-1.69)$ & $1.08(0.84-1.41)$ & $0.87(0.49-1.54)$ & $0.75(0.53-1.04)$ & $0.79(0.53-1.19)$ \\
\hline \multicolumn{8}{|l|}{ ERI (low) } \\
\hline ERI (med) & $2.81(1.72-4.57)^{* *}$ & $2.10(1.15-3.82)^{*}$ & $1.97(1.43-2.72)^{* *}$ & $1.56(1.12-2.17)^{*}$ & $3.29(1.93-5.63)^{* *}$ & $2.08(1.33-3.24)^{* *}$ & $1.93(1.25-2.97)^{* *}$ \\
\hline \multirow[t]{2}{*}{ ERI (high) } & $5.65(3.32-9.62)^{* *}$ & $6.85(3.75-12.50)^{* *}$ & $5.62(3.94-8.00)^{* *}$ & $4.07(2.94-5.63)^{* *}$ & $7.76(3.21-18.73)^{* *}$ & $5.72(3.75-8.74)^{* *}$ & $3.09(2.01-4.76)^{* *}$ \\
\hline & \multicolumn{3}{|c|}{ Nursing homes $(n=208)$ Nursing homes $(n=345)$ Nursing homes $(n=397)$} & Nursing homes $(n=124)$ & Nursing homes $(n=673)$ & Nursing homes $(n=138)$ & Nursing homes $(n=254)$ \\
\hline Qualification (aids) & $2.27(0.86-6.01)$ & $1.54(0.74-3.21)$ & $1.92(0.87-4.25)$ & (only RN in Sample) & $2.65(1.07-6.56)^{*}$ & (only RN in Sample) & $3.85(1.58-9.37)^{* *}$ \\
\hline \multicolumn{8}{|l|}{ L\&B (low) } \\
\hline L\&B (medium) & $0.49(0.12-2.00)$ & $1.59(0.64-3.92)$ & $0.86(0.36-2.07)$ & $1.63(0.38-6.96)$ & $1.73(0.56-5.39)$ & $0.76(0.15-3.93)$ & $1.13(0.46-2.75)$ \\
\hline L\&B (high) & $1.71(0.58-5.05)$ & $0.95(0.39-2.33)$ & $3.04(1.30-7.13)^{*}$ & $0.38(0.06-2.57)$ & $1.37(0.37-4.98)$ & $7.84(1.53-40.09)^{*}$ & $1.37(0.58-3.23)$ \\
\hline \multicolumn{8}{|c|}{ Lifting aids (available + used) } \\
\hline Lifting aids $(\mathrm{n} / \mathrm{a})$ & $2.43(0.32-18.20)$ & $1.45(0.57-3.73)$ & $2.95(1.38-6.30) *$ & $0.60(0.13-2.85)$ & $1.17(0.14-10.11)$ & $12.91(1.12-148.19)^{*}$ & $0.97(0.44-2.13)$ \\
\hline
\end{tabular}




\begin{tabular}{|c|c|c|c|c|c|c|c|}
\hline & Belgium & Germany & France & Italy & Netherlands & Poland & Slovakia \\
\hline & Hospital $(n=1819)$ & Hospital $(n=2181)$ & Hospital $(n=3043)$ & Hospital $(n=3478)$ & Hospital $(n=2338)$ & Hospital $(n=2520)$ & Hospital $(n=1391)$ \\
\hline & Odds $(95 \% \mathrm{CI})$ & Odds $(95 \% \mathrm{CI})$ & Odds $(95 \% \mathrm{CI})$ & Odds $(95 \% \mathrm{CI})$ & Odds $(95 \% \mathrm{CI})$ & Odds $(95 \% \mathrm{CI})$ & Odds $(95 \% \mathrm{CI})$ \\
\hline \multicolumn{8}{|c|}{ Quantitative demands (low) } \\
\hline Quantitative demands (med) & $1.99(0.43-9.20)$ & $4.29(1.39-13.26)^{*}$ & $0.67(0.28-1.60)$ & $1.68(0.41-6.86)$ & $1.67(0.62-4.48)$ & $0.83(0.19-3.76)$ & $1.64(0.71-3.78)$ \\
\hline \multicolumn{8}{|c|}{ Influence at work (high) } \\
\hline Influence at work (med) & $1.43(0.43-4.70)$ & $1.15(0.53-2.52)$ & $0.72(0.32-1.58)$ & $1.10(0.27-4.56)$ & $1.95(0.69-5.52)$ & $0.99-(0.22-4.50)$ & $0.74(0.30-1.82)$ \\
\hline \multicolumn{8}{|l|}{ ERI (low) } \\
\hline ERI (med) & $15.71(3.23-76.46)^{* *}$ & $3.16(0.36-27.9)$ & $1.43(0.51-3.97)$ & $0.83(0.06-11.63)$ & $1.88(0.75-4.71)$ & - & $1.64(0.65-4.17)$ \\
\hline \multirow[t]{2}{*}{ ERI (high) } & $3.54(0.81-15.48)$ & $17.45(2.02-150.46)^{*}$ & $5.92(2.01-17.42)^{* *}$ & $9.42(0.91-97.53)$ & $2.32(0.44-12.32)$ & \multicolumn{2}{|c|}{$2.1 \mathrm{E}+09(3.1 \mathrm{E}+08-1.2 \mathrm{E}+10)^{* *} 3.30(1.17-9.31)^{*}$} \\
\hline & Home care $(n=1253)$ & Home care $(n=256)$ & Home care $(n=43)$ & Home care $(n=285)$ & Home care $(n=572)$ & Home care $(n=161)$ & Home care $(n=33)$ \\
\hline \multicolumn{8}{|l|}{ L\&B (low) } \\
\hline L\&B (medium) & $2.06(0.69-6.11)$ & $3.33(1.05-10.56)^{*}$ & $\mathrm{n} / \mathrm{a}$ & $0.58(0.14-2.41)$ & $0.69(0.27-1.73)$ & (To view cases) & $\mathrm{n} / \mathrm{a}$ \\
\hline L\&B (high) & $1.93(0.65-5.69)$ & $5.65(1.81-17.60)^{* *}$ & $\mathrm{n} / \mathrm{a}$ & $0.73(0.13-4.19)$ & $0.15(0.02-1.32)$ & (To view cases) & $\mathrm{n} / \mathrm{a}$ \\
\hline \multicolumn{8}{|c|}{ Lifting aids (available + used) } \\
\hline Lifting aids $(\mathrm{n} / \mathrm{a})$ & $1.17(0.67-2.01)$ & $0.43(0.19-0.99)$ & $\mathrm{n} / \mathrm{a}$ & $0.45(0.15-1.36)$ & $1.29(0.50-3.33)$ & (to view cases) & \\
\hline $\begin{array}{l}\text { Lifting aids (not used) } \\
\text { Ouantitative demands (low) }\end{array}$ & $0.23(0.03-1.76)$ & $0.79(0.21-2.93)$ & $\mathrm{n} / \mathrm{a}$ & $0.22(0.02-2.27)$ & $\mathrm{n} / \mathrm{a}$ & (to view cases) & $\mathrm{n} / \mathrm{a}$ \\
\hline Quantitative demands (med) & $1.00(0.44-2.26)$ & $1.09(0.33-3.57)$ & $\mathrm{n} / \mathrm{a}$ & $1.27(0.30-5.30)$ & $1.33(0.49-3.61)$ & $1.39(0.28-6.92)$ & $\mathrm{n} / \mathrm{a}$ \\
\hline Quantitative demands (high) & $1.86(0.89-3.90)$ & $1.87(0.66-5.33)$ & $\mathrm{n} / \mathrm{a}$ & $1.91(0.71-5.14)$ & $3.07(1.10-8.58)^{*}$ & $1.44(0.24-8.85)$ & $\mathrm{n} / \mathrm{a}$ \\
\hline \multicolumn{8}{|c|}{ Influence at work (high) } \\
\hline Influence at work (med) & $1.13(0.65-1.94)$ & $0.63(0.20-1.99)$ & $\mathrm{n} / \mathrm{a}$ & $0.45(0.15-1.38)$ & $0.84(0.28-2.51)$ & $0.32(0.05-1.99)$ & $\mathrm{n} / \mathrm{a}$ \\
\hline Influence at work (low) & $0.76(0.39-1.50)$ & $0.49(0.18-1.40)$ & $\mathrm{n} / \mathrm{a}$ & $0.63(0.22-1.82)$ & $1.59(0.52-4.87)$ & $0.18(0.02-1.82)$ & $\mathrm{n} / \mathrm{a}$ \\
\hline \multicolumn{8}{|l|}{ ERI (low) } \\
\hline ERI (med) & $3.54(1.69-7.43)^{* *}$ & $1.14(0.12-10.94)$ & $\mathrm{n} / \mathrm{a}$ & $0.38(0.08-1.76)$ & $3.71(1.37-10.02)^{*}$ & $2.13(0.17-27.31)$ & $\mathrm{n} / \mathrm{a}$ \\
\hline ERI (high) & $3.76(1.70-8.34)^{* * *}$ & $7.75(0.87-68.75)$ & $\mathrm{n} / \mathrm{a}$ & $3.67(1.11-12.16)^{*}$ & $10.74(3.57-32.33)^{* * *}$ & $6.89(0.73-64.75)$ & $\mathrm{n} / \mathrm{a}$ \\
\hline
\end{tabular}

${ }^{*} p<0.05,{ }^{* *} p<0.01$. 


\section{Acknowledgements}

NEXT was funded by the European Commission within the 5th framework programme (EU-project no.: QLK6-CT-2001-00475). This secondary analysis was funded by the Berufsgenossenschaft für Gesundheitsdienst und Wohlfahrtspflege, BGW (Germany).

\section{References}

Armstrong, T.J., Buckle, P., Fine, L.J., Hagberg, M., Jonsson, B., Kilbom, A., Kuorinka, I.A., Silverstein, B.A., Sjogaard, G., Viikari-Juntura, E.R., 1993. A conceptual model for work-related neck and upper-limb musculoskeletal disorders. Scandinavian Journal of Work Environment \& Health 19 (2), 73-84.

Bongers, P.M., de Winter, C.R., Kompier, M.A., Hildebrandt, V.H., 1993. Psychosocial factors at work and musculoskeletal disease. Scandinavian Journal of Work Environment \& Health 19 (5), 297-312.

Carayon, P., Smith, M.J., Haims, M.C., 1999. Work organization, job stress, and work-related musculoskeletal disorders. Human Factors 41 (4), 644-663.

Cheung, K., Gillen, M., Faucett, J., N., K., 2006. The Prevalence of and Risk Factors for Back Pain Among Home Care Nursing Personnel in Hong Kong. American Journal of Industrial Medicine 49 (1), 14-22.

Crook, J., Milner, R., Schultz, I.Z., Stringer, B., 2002. Determinants of occupational disability following a low back injury: a critical review of the literature. Journal of Occupational Rehabilitation 12 (4), 277-295.

Davis, K.G., Heaney, C.A., 2000. The relationship between psychosocial work characteristics and low back pain: underlying methodological issues. Clinical Biomechanics 15 (6), 289-406.

Elders, L.A.M., Burdorf, A., 2001. Interrelations of risk factors and low back pain in scaffolders. Occupational and Environmental Medicine 58 (9), 597-603.

Grabbe, Y., Nolting, H.-D., Loos, S., 2005. DAK-BGW Gesundheitsreport 2005-Stationäre Krankenpflege 2005. BGW, DAK, Hamburg.

Gunnarsdottir, H.K., Rafnsdottir, G.L., Helgadottir, B., Tomasson, K., 2003. Psychosocial risk factors for musculoskeletal symptoms among women working in geriatric care. American Journal of Industrial Medicine 44 (6), 679-684.

Hasselhorn, H.-M., Tackenberg, P., Müller, B.H., 2003. Working Conditions and Intent to Leave the Profession Among Nursing Staff in Europe. National Institute for Working Life, Stockholm.

Hasselhorn, H.-M., Müller, B.H., Tackenberg, P., 2005. NEXT Scientific Report July 2005. http://www.next.uni-wuppertal.de/download/NEXTscientificreportjuly2005.pdf, Wuppertal. [last access: 2006-11-13]

Hignett, S., 2003. Intervention strategies to reduce musculoskeletal injuries associated with handling patients: a systematic review. Occupational and Environmental Medicine 60(9), E6. nicht im Text
Hoogendoorn, W.E., Bongers, P.M., de Vet, H.C., Ariens, G.A., van Mechelen, W., Bouter, L.M., 2002. High physical work load and low job satisfaction increase the risk of sickness absence due to low back pain: results of a prospective cohort study. Occupational and Environmental Medicine 59 (5), 323-328.

Ijzelenberg, W., Burdorf, A., 2004. Patterns of care for low back pain in a working population. Spine 29 (12), 1362-1368.

Kristensen, T.S., Hannerz, H., Hogh, A., Borg, V., 2005. The Copenhagen Psychosocial Questionnaire: a tool for the assessment and improvement of the psychosocial work environment. Scandinavian Journal of Work Environment \& Health 31 (6), 438-449.

Kümmerling, A., Hasselhorn, H.-M., Tackenberg, P., 2003. Psychometric properties of the scales used in the NEXTStudy. In: Hasselhorn, H.-M., Tackenberg, P., Müller, B.H. (Eds.), Working Conditions and Intent to Leave the Profession Among Nursing Staff in Europe. National Institute for Working Life and authors, Stockholm, pp. 237-257.

Lagerstrom, M., Hansson, T., Hagberg, M., 1998. Workrelated low-back problems in nursing. Scandinavian Journal of Work Environment \& Health 24 (6), 449-464.

Linton, S., 2000. Psychological risk factors for neck and back pain. In: Nachemson, A.L., Jonsson, E. (Eds.), Neck and Back Pain: The Scientific Evidence of Causes, Diagnosis, and Treatment. Lippincott Williams \& Wilkins, Philadelphia, pp. 57-78.

Menzel, N.N., 2004. Back pain prevalence in nursing personnel: measurement issues. AAOHN Journal 52 (2), 54-65.

Panel on Musculoskeletal Disorders and the WorkplaceCommission on Behavioral and Social Sciences and Education-National Research Council, 2001. Musculoskeletal Disorders and the Workplace: Low Back and Upper Extremities. National Academy Press, Washington, DC.

Paoli, P., Merllié, D., 2001. Third European Survey on Working Conditions 2000. European Foundation for the Improvement of Living and Working Conditions, Dublin.

Pattani, S., Constantinovici, N., Williams, S., 2001. Who retires early from the NHS because of ill health and what does it cost? A national cross sectional study. British Medical Journal 322 (7280), 208-209.

Rugulies, R., Braff, J., Frank, J.W., Aust, B., Gillen, M., Yen, I.H., Bhatia, R., Ames, G., Gordon, D.R., Janowitz, I., Oman, D., Jacobs, B.P., Blanc, P., 2004. The psychosocial work environment and musculoskeletal disorders: design of a comprehensive interviewer-administered questionnaire. American Journal of Industrial Medicine 45 (5), 428-439.

Sherehiy, B., Karwowski, W., Marek, T., 2004. Relationship between risk factors and musculoskeletal disorders in the nursing profession: a systematic review. Occupational Ergonomics 4, 241-279.

Siegrist, J., 1996. Adverse health effects of high-effort/lowreward conditions. Journal of Occupational Health Psychology 1 (1), 27-41.

Siegrist, J., Starke, D., Chandola, T., Godin, I., Marmot, M., Niedhammer, I., Peter, R., 2004. The measurement of effort-reward imbalance at work: European comparisons. Social Science \& Medicine 58 (8), 1483-1499. 
Simon, M., Hasselhorn, H.-M., Kümmerling, A., 2005. Physical exposure to lifting and bending tasks among nurses in Europe. In: NEXT Scientific Report July 2005. http://www.next.uni-wuppertal.de/download/NEXTscientificreportjuly2005.pdf, Wuppertal. [last access: 2006-11-13]

Smedley, J., Trevelyan, F., Inskip, H., Buckle, P., Cooper, C., Coggon, D., 2003. Impact of ergonomic intervention on back pain among nurses. Scandinavian Journal of Work Environment \& Health 29 (2), 117-123.

SPSS Inc., 2005. SPSS 11.04 for Macintosh. Chicago.

Theorell, T., Perski, A., Akerstedt, T., Sigala, F., Ahlberg-Hulten, G., Svensson, J., Eneroth, P., 1988. Changes in job strain in relation to changes in physiological state. A longitudinal study. Scandinavian Journal of Work Environment \& Health 14 (3), 189-196.

Turner, J.A., Franklin, G., Heagerty, P.J., Wu, R., Egan, K., Fulton-Kehoe, D., Gluck, J.V., Wickizer, T.M., 2004. The association between pain and disability. Pain 112 (3), 307-314.

van den Heuvel, S.G., Ariens, G.A.M., Boshuizen, H.C., Hoogendoom, W.E., Bongers, P.M., 2004. Prognostic factors related to recurrent low-back pain and sickness absence. Scandinavian Journal of Work Environment \& Health 30 (6), 459-467.

van Vegchel, N., de Jonge, J., Bosma, H., Schaufeli, W., 2005. Reviewing the effort-reward imbalance model: drawing up the balance of 45 empirical studies. Social Science \& Medicine 60 (5), 1117-1131.

Violante, F.S., Fiori, M., Fiorentini, C., Risi, A., Garagnani, G., Bonfiglioli, R., Mattioli, S., 2004. Associations of psychosocial and individual factors with three different categories of back disorder among nursing staff. Journal of Occupational Health 46 (2), 100-108.

von Korff, M., Ormel, J., Keefe, F.J., Dworkin, S.F., 1992. Grading the severity of chronic pain. Pain 50 (2), 133-149. 